\title{
Metabolic syndrome, insulin resistance and kidney function in non-diabetic individuals
}

\author{
Barry R. Johns ${ }^{1}$, Alan C. Pao ${ }^{2}$ and Sun H. Kim ${ }^{1}$ \\ ${ }^{1}$ Division of Endocrinology, Gerontology, and Metabolism, Department of Medicine, Stanford University School of Medicine, Stanford, \\ CA, USA and ${ }^{2}$ Division of Nephrology, Department of Medicine, Stanford University School of Medicine, Stanford, CA, USA \\ Correspondence and offprint requests to: Barry R. Johns; E-mail: barryrjohns@gmail.com
}

\begin{abstract}
Background. Metabolic syndrome has been recently identified as a risk factor for chronic kidney disease (CKD). Since the five individual components of the metabolic syndrome have also been identified as risk factors for CKD, the metabolic syndrome diagnosis may represent an aggregate of CKD risk factors. On the other hand, the components of the metabolic syndrome are also associated with insulin resistance, which may directly mediate the increased CKD risk. Methods. This study was a cross-sectional evaluation of the relationship between metabolic syndrome, insulin resistance and estimated glomerular filtration rate (eGFR) in 574 non-diabetic individuals. Insulin resistance was directly quantified using the insulin suppression test, and the metabolic syndrome components were measured. eGFR was calculated using the three validated estimation equations: the Chronic Kidney Disease Epidemiology Collaboration equation, the Mayo quadratic equation and the Modification of Diet in Renal Disease study equation.

Results. While CKD prevalence was higher and mean eGFR was lower in individuals who met the metabolic syndrome criteria compared with those who did not, we did not observe a significant relationship between insulin resistance and eGFR. Of all of the components of the metabolic syndrome, only hypertension was significantly associated with CKD prevalence [odds ratio (95\% confidence interval), 3.5 (1.2-10.1), $\mathrm{P}=0.02]$.

Conclusion. Although CKD is more common among individuals with the metabolic syndrome, insulin resistance is not a common factor.
\end{abstract}

Keywords: chronic kidney disease; estimated glomerular filtration rate; insulin resistance; metabolic syndrome

\section{Introduction}

Recent studies have suggested that individuals with metabolic syndrome are at increased risk for developing chronic kidney disease (CKD) [1-4]. The mechanism behind this increased risk may be due to the aggregation of known risk factors for CKD in the metabolic syndrome diagnosis. On the other hand, the metabolic syndrome diagnosis may indicate the presence of insulin resistance, which may directly increase the risk for CKD. A few studies have suggested a link between insulin resistance and $\mathrm{CKD}[2,5,6]$; however, these studies used surrogate measures of insulin resistance based on fasting insulin concentration. Unfortunately, fasting insulin concentration captures $<40 \%$ of the variability in insulin resistance $[7,8]$, indicating that insulin concentration is a less precise estimate of insulin action.

The few studies that have used direct measures of insulin action to evaluate the relationship between insulin resistance and kidney function were conducted in individuals with pre-existing kidney disease [9-11]. Although a relationship was identified, it is not clear whether declining kidney function engenders insulin resistance or whether insulin resistance predisposes to declining kidney function.

To better understand the relationship between kidney function and insulin resistance, we evaluated the association between estimates of glomerular filtration rate (eGFR) and a direct measure of insulin resistance, using the insulin suppression test, in 574 relatively healthy individuals without diabetes or known kidney disease. The purpose was to evaluate the association between kidney function and insulin resistance in a population prior to overt kidney disease. We concurrently evaluated the impact of the diagnosis of metabolic syndrome on eGFR. We hypothesized that although the diagnosis of metabolic syndrome would be associated with lower eGFR, with direct measurement of insulin resistance, there would be no association between insulin resistance and eGFR.

\section{Materials and methods}

The study population consisted of 574 non-diabetic individuals (348 women and 226 men) who have been enrolled for our cross-sectional study of insulin resistance since 2000. The study was approved by Stanford University's Internal Review Board and was in compliance with the principles outlined in the Declaration of Helsinki. All subjects provided written informed consent for participation. All subjects were generally healthy and had the following 
procedures on the same day: measurement of height, weight, waist circumference, blood pressure, lipid assessment, serum creatinine and insulin suppression test to measure insulin sensitivity.

All procedures were performed in the Clinical and Translational Research Unit after $12 \mathrm{~h}$ of fasting. Blood pressure was measured using a Dinamap automatic blood pressure recorder (GE Healthcare, Tampa, FL). Before the blood pressure measurements, patients were seated quietly in a chair for 5 min with their feet on the floor and one arm supported at heart level. Using an appropriately sized cuff, three blood pressure readings were taken at 1-min intervals, and the mean of these readings was used for data analysis. Waist circumference was measured by placing a measuring tape around the waist at the upper point of the iliac crest and determined during minimal inspiration as previously described [12].

Lipid panel and serum creatinine were measured in the core laboratory at Stanford. As our population was generally healthy, we used two eGFR estimations recently developed for the general population including the Mayo Clinic Quadratic formula [13] and the Chronic Kidney Disease Epidemiology (CKD-EPI) Collaboration equation [14]. For comparison, prevalence of CKD was also calculated using the traditional Modification of Diet in Renal Disease (MDRD) study equation (abstract by Levey et al., J Am Soc Nephrol 2000). CKD was defined as having an eGFR $<60 \mathrm{~mL} / \mathrm{min}$.

Insulin sensitivity was directly measured using a modified version [15] of the insulin suppression test. Insulin sensitivity measurements from the insulin suppression test are highly correlated with results from the hyperinsulinemic euglycemic clamp $(r=0.93)$ [16]. Following an overnight fast, an intravenous catheter was placed in each of the subjects' arms. One arm was used for the administration of a 180-min infusion of octreotide $\left(0.27 \mu \mathrm{g} / \mathrm{m}^{2} / \mathrm{min}\right)$, insulin $\left(32 \mathrm{mU} / \mathrm{m}^{2} / \mathrm{min}\right)$ and glucose $\left(267 \mathrm{mg} / \mathrm{m}^{2} / \mathrm{min}\right)$; the other arm was used for drawing blood. Plasma glucose and insulin concentrations were measured every $10 \mathrm{~min}$ from 150 to $180 \mathrm{~min}$ and averaged to obtain the steady-state plasma glucose (SSPG) and insulin concentrations. Since steady-state plasma insulin concentrations are comparable in all individuals and glucose infusion rate is identical, the SSPG concentration provides a direct measure of insulin resistance; the higher the SSPG concentration, the more insulin resistant the individual. To evaluate the relationship between insulin resistance and kidney function, subjects were grouped into tertiles based on SSPG concentrations, with the most insulin-sensitive individuals in Tertile 1 and the most insulin-resistant individuals in Tertile 3. Glucose concentrations were measured by the oxidase method (Beckman Analyzer 2, Brea, CA).

For the diagnosis of metabolic syndrome, we used the 2001 ATP III definition [17], with modifications as outlined in the 2005 statement from the American Heart Association/National Heart, Lung and Blood Institute [18]. The five components of the metabolic syndrome are as follows: (i) waist circumference $>102 \mathrm{~cm}$ in men or $>88 \mathrm{~cm}$ in women; (ii) blood pressure $>130 / 85 \mathrm{mmHg}$ or on drug treatment for hypertension; (iii) serum triglycerides $\geq 1.7 \mathrm{mmol} / \mathrm{L}$ or on drug treatment for hypertriglyceridemia, (iv) serum high-density lipoprotein cholesterol (HDL-C) $<1.03$ $\mathrm{mmol} / \mathrm{L}$ in men and $<1.3 \mathrm{mmol} / \mathrm{L}$ in women or on drug treatment for low HDL-C and (v) fasting plasma glucose $\geq 5.6 \mathrm{mmol} / \mathrm{L}$. Metabolic syndrome is considered to be present if any three of the five traits are present.

Statistical analysis was performed using SPSS (version 17 for Windows; SPSS, Chicago, IL). All data are median (interquartile range) unless otherwise specified. Analysis of variance or chi-square tests for categorical variables were used to compare clinical and laboratory characteristics. A linear model, adjusted for age, gender and ethnicity, was used to evaluate the relationship between insulin resistance (SSPG) and eGFR. In a second model, we also adjusted for use of medications that may prevent or slow progression of kidney disease, including angiotensin-converting enzyme (ACE) inhibitors $[19,20]$ or angiotensin receptor blockers (ARB) [21, 22] and 3-hydroxy-3methyl-glutaryl-CoA reductase inhibitors (statins) [23, 24]. Variables that were not normally distributed were log transformed.

\section{Results}

We first categorized our study population by diagnosis of metabolic syndrome (Table 1). Overall, $42 \%$ of the study population met the criteria for metabolic syndrome. Individuals meeting this criteria were older, more likely to be male and heavier compared with individuals who did not. As expected, all components of the metabolic syndrome were more unfavorable in those who met the metabolic syndrome criteria. In addition, individuals with metabolic

Table 1. Characteristics of individuals with and without metabolic syndrome

\begin{tabular}{|c|c|c|c|}
\hline \multirow[b]{2}{*}{ Variables } & \multicolumn{2}{|c|}{ Metabolic syndrome } & \multirow[b]{2}{*}{$\mathrm{P}$} \\
\hline & Absent & Present & \\
\hline$N$ & 334 & 240 & \\
\hline Age (years) & $50(43-55)$ & $53(45-59)$ & $<0.001$ \\
\hline Body mass index $\left(\mathrm{kg} / \mathrm{m}^{2}\right)$ & $27.7(25.3-30.8)$ & $31.6(29.3-34.8)$ & $<0.001$ \\
\hline Men $(\%)$ & 35.3 & 45.0 & 0.019 \\
\hline Non-Hispanic White (\%) & 65.3 & 65.8 & 0.89 \\
\hline \multicolumn{4}{|l|}{ Metabolic syndrome components } \\
\hline Waist circumference $(\mathrm{cm})$ & $93(87-101)$ & $106(98-113)$ & $<0.001$ \\
\hline Systolic blood pressure (mmHg) & $116(109-126)$ & $131(117-140)$ & $<0.001$ \\
\hline Diastolic blood pressure ( $\mathrm{mmHg}$ ) & $71(66-78)$ & $75(69-83)$ & $<0.001$ \\
\hline Plasma glucose $(\mathrm{mmol} / \mathrm{L})$ & $5.2(4.9-5.4)$ & $5.6(5.3-6.1)$ & $<0.001$ \\
\hline Triglycerides $(\mathrm{mmol} / \mathrm{L})$ & $0.99(0.71-1.33)$ & $1.73(1.18-2.29)$ & $<0.001$ \\
\hline HDL-C (mmol/L) & $1.30(1.06-1.56)$ & $1.01(0.88-1.21)$ & $<0.001$ \\
\hline \multicolumn{4}{|l|}{ Insulin resistance } \\
\hline \multicolumn{4}{|l|}{ Medications } \\
\hline Use of ACE inhibitor or ARB (\%) & 5.4 & 22.5 & $<0.001$ \\
\hline Use of statin $(\%)$ & 9.3 & 15.4 & 0.03 \\
\hline \multicolumn{4}{|l|}{ Kidney function estimates } \\
\hline Serum creatinine $(\mu \mathrm{mol} / \mathrm{L})$ & $65.2(54.0-76.3)$ & $68.6(60.2-76.3)$ & 0.03 \\
\hline eGFR, Mayo $\left(\mathrm{mL} / \mathrm{min} / 1.73 \mathrm{~m}^{2}\right)$ & $101.7(96.2-110.9)$ & $100.2(93.3-110.3)$ & 0.07 \\
\hline eGFR, CKD-EPI (mL/min/1.73m²) & $89.3(78.5-100.9)$ & $87.5(75.9-96.7)$ & 0.06 \\
\hline $\mathrm{eGFR}, \mathrm{MDRD}\left(\mathrm{mL} / \mathrm{min} / 1.73 \mathrm{~m}^{2}\right)$ & $84.4(78.5-100.9)$ & $83.7(73.5-94.2)$ & 0.36 \\
\hline CKD, Mayo (\%) & 0.3 & 0.8 & 0.74 \\
\hline CKD, CKD-EPI (\%) & 2.4 & 5.8 & 0.03 \\
\hline CKD, MDRD (\%) & 3.0 & 5.8 & 0.09 \\
\hline
\end{tabular}

${ }^{\mathrm{a}}$ Values represent median (interquartile range) unless otherwise specified. 
syndrome had higher degrees of insulin resistance as quantified by SSPG concentration. Finally, individuals with metabolic syndrome were more likely to be treated with ACE inhibitors, ARBs or statins.

The prevalence of CKD was generally low in the study population but varied greatly depending on the eGFR calculation. We calculated the overall prevalence of CKD to be $0.7 \%$ (4/574) with the Mayo Quadratic equation, 3.8\% (22/574) with the CKD-EPI equation and 4.2\% (24/574) with the traditional MDRD equation. Despite differences in the prevalence of CKD, estimates of kidney function tended to be lower in those with metabolic syndrome compared with those without the syndrome (Table 1). For example, serum creatinine was significantly higher, and CKD prevalence was $\geq 2$-fold in those with the metabolic syndrome compared with those without the diagnosis. The difference in CKD prevalence was only significant when eGFR was calculated using the CKD-EPI equation.

Of all of the components of the metabolic syndrome, hypertension was most strongly associated with CKD. Using the CKD-EPI equation to calculate eGFR, we found a 4-fold increase in the prevalence of CKD in individuals with hypertension compared with those without hypertension ( 6.5 versus $1.6 \%, \mathrm{P}=0.002)$. Similar trends were obtained when eGFR was calculated via the Mayo ( 1.1 versus $0.3 \%, \mathrm{P}=0.34)$ and MDRD (6.9 versus $1.9 \%, \mathrm{P}=0.005)$ equations. Hypertension remained a significant predictor of $\mathrm{CKD}$ in a logistic regression model even when adjusted for age, gender and ethnicity [odds ratio 3.5 (95\% confidence interval, 1.2-10.1), $\mathrm{P}=0.02$ using CKD-EPI and 3.2 (1.2-8.7), $\mathrm{P}=0.02$ using MDRD]. In contrast, none of the other metabolic syndrome components was significantly associated with the presence of CKD.

To determine the impact of insulin resistance on kidney function, we also categorized our study population by tertiles of insulin resistance (Table 2) and evaluated the relationship between insulin resistance and baseline characteristics. As the degree of insulin resistance increased, body mass index also increased. In terms of ethnicity, the prevalence of non-Hispanic Whites significantly decreased with increasing degrees of insulin resistance. Age and gender were similar across the three tertiles.

As expected, all components of metabolic syndrome became more unfavorable with increasing degrees of insulin resistance. Therefore, the prevalence of metabolic syndrome (three or more components) also increased with increasing degrees of insulin resistance. However, there were discrepancies between the diagnosis of metabolic syndrome and the degree of insulin resistance. For example, $16 \%$ of the most insulin-sensitive individuals (Tertile 1) actually met the criteria for metabolic syndrome. Conversely, $30 \%$ of the most insulin-resistant individuals failed to meet the criteria for metabolic syndrome. These findings highlight the limitations of using the metabolic syndrome diagnosis as a surrogate measure for insulin resistance.

With regard to kidney function, there was no relationship between insulin resistance and eGFR. Although all individuals with CKD defined by the Mayo Quadratic equation were categorized into the most insulin-resistant tertile, this group consisted of only four individuals. We also did not observe a relationship between insulin resistance and eGFR calculated by the other two equations.

Figure 1 illustrates the lack of relationship between SSPG concentration and eGFR calculated with the Mayo Quadratic formula (A) and CKD-EPI (B). Although there was a 6-fold difference in SSPG concentration among individuals, there was no obvious relationship between insulin resistance and eGFR calculated with either equation. Moreover, in a linear model, there was no significant relationship between SSPG

Table 2. Characteristics of individuals by tertile of insulin resistance ${ }^{a}$

\begin{tabular}{|c|c|c|c|c|}
\hline & Insulin sensitive SSPG 1 & Insulin intermediate SSPG 2 & Insulin resistant SSPG 3 & $\mathrm{P}$ \\
\hline$N$ & 193 & 189 & 192 & \\
\hline SSPG & $4.2(3.4-5.1)$ & $8.2(7.1-9.3)$ & $13.0(11.7-14.5)$ & $<0.001$ \\
\hline Age (years) & $51(45-57)$ & $51(42-57)$ & $51(42-57)$ & 0.57 \\
\hline Body mass index $\left(\mathrm{kg} / \mathrm{m}^{2}\right)$ & $26.9(24.3-28.9)$ & $29.9(27.0-32.7)$ & $33.2(29.3-35.2)$ & $<0.001$ \\
\hline Men $(\%)$ & 37.8 & 38.6 & 41.7 & 0.72 \\
\hline Non-Hispanic White (\%) & 75.1 & 63.5 & 57.8 & 0.001 \\
\hline \multicolumn{5}{|l|}{ Metabolic syndrome components } \\
\hline Waist circumference $(\mathrm{cm})$ & $91(84-99)$ & $100(92-109)$ & $105(97-113)$ & $<0.001$ \\
\hline Systolic blood pressure (mmHg) & $116(108-130)$ & $121(111-133)$ & $126(114-137)$ & $<0.001$ \\
\hline Diastolic blood pressure $(\mathrm{mmHg})$ & $72(66-78)$ & $73(67-80)$ & $74(69-81)$ & 0.05 \\
\hline Plasma glucose $(\mathrm{mmol} / \mathrm{L})$ & $5.1(4.8-5.4)$ & $5.3(5.1-5.6)$ & $5.6(5.2-6.0)$ & $<0.001$ \\
\hline Triglycerides $(\mathrm{mmol} / \mathrm{L})$ & $0.90(0.68-1.37)$ & $1.20(0.86-1.70)$ & $1.53(1.13-2.27)$ & $<0.001$ \\
\hline $\mathrm{HDL}-\mathrm{C}(\mathrm{mmol} / \mathrm{L})$ & $1.30(1.06-1.62)$ & $1.17(0.98-1.40)$ & $1.04(0.91-1.24)$ & $<0.001$ \\
\hline Metabolic syndrome (\%) & 15.5 & 39.7 & 71.9 & $<0.001$ \\
\hline \multicolumn{5}{|l|}{ Medications } \\
\hline Use of ACE inhibitor or ARB (\%) & 8.8 & 10.1 & 18.8 & 0.006 \\
\hline Use of statin $(\%)$ & 9.3 & 12.2 & 14.1 & 0.35 \\
\hline \multicolumn{5}{|l|}{ Kidney function estimates } \\
\hline Serum creatinine $(\mu \mathrm{mol} / \mathrm{L})$ & $68.6(57.6-76.3)$ & $67.1(57.2-76.3)$ & $67.9(55.1-76.3)$ & 0.93 \\
\hline eGFR, Mayo $\left(\mathrm{mL} / \mathrm{min} / 1.73 \mathrm{~m}^{2}\right)$ & $100.9(95.5-109.1)$ & $101.6(94.5-110.5)$ & $101.8(95.5-111.8)$ & 0.56 \\
\hline eGFR, CKD-EPI (mL/min/1.73m $\left.{ }^{2}\right)$ & $88.0(74.2-99.1)$ & $89.8(78.3-98.6)$ & $89.0(76.5-100.7)$ & 0.48 \\
\hline $\mathrm{eGFR}, \operatorname{MDRD}\left(\mathrm{mL} / \mathrm{min} / 1.73 \mathrm{~m}^{2}\right)$ & $83.7(72.9-95.1)$ & $84.2(76.2-95.0)$ & $84.4(74.2-96.7)$ & 0.44 \\
\hline CKD, Mayo (\%) & 0 & 0 & 2.1 & 0.02 \\
\hline CKD, CKD-EPI (\%) & 4.7 & 3.2 & 3.6 & 0.74 \\
\hline CKD, MDRD (\%) & 5.7 & 3.2 & 3.6 & 0.42 \\
\hline
\end{tabular}

${ }^{\mathrm{a}}$ Values represent median (interquartile range) unless otherwise specified. 

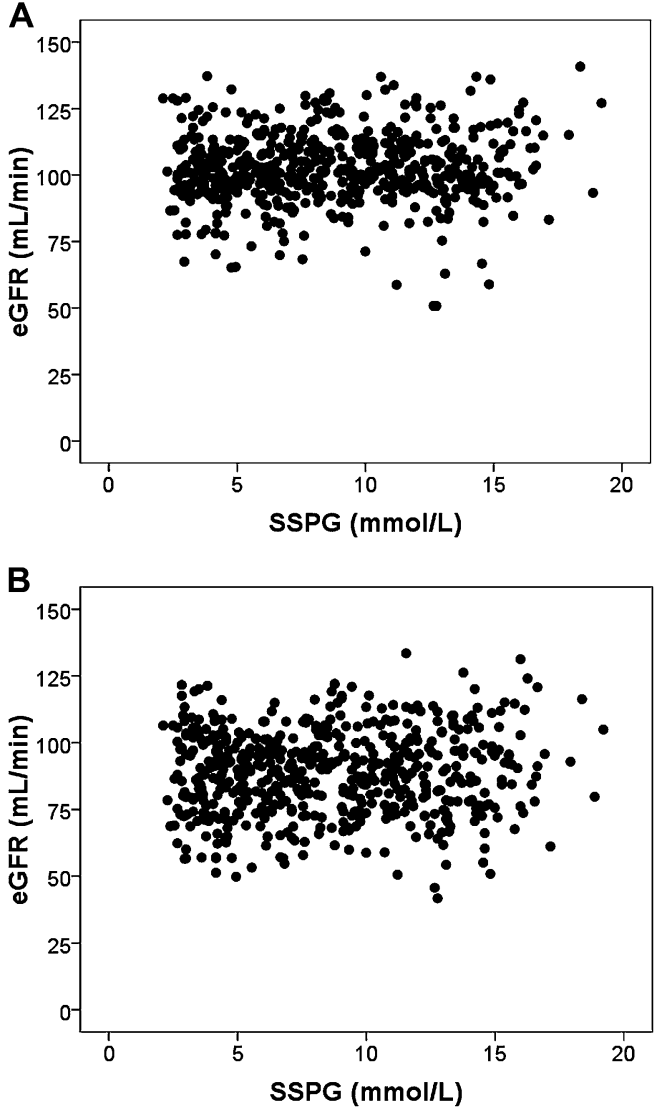

Fig. 1. (A) The relationship between $\mathrm{SSPG}$ concentration $(\mathrm{mmol} / \mathrm{L})$ and eGFR ( $\mathrm{mL} / \mathrm{min}$ ) as determined by the Mayo quadratic formula. (B) The relationship between SSPG concentration $(\mathrm{mmol} / \mathrm{L})$ and eGFR $(\mathrm{mL} / \mathrm{min})$ as determined by the CKD-EPI formula.

concentration and eGFR even after adjustment for age, gender and ethnicity $(\mathrm{P}=0.72$ for CKD-EPI, $\mathrm{P}=0.72$ for Mayo and $\mathrm{P}=0.58$ for MDRD). Further adjustment for treatment of individuals with ACE inhibitors/ARBs or statins did not alter the relationship between SSPG concentration and eGFR ( $\mathrm{P}=0.40$ for CKD-EPI, $\mathrm{P}=0.29$ for Mayo and $\mathrm{P}=0.32$ for $\mathrm{MDRD})$.

Since prior publications examining the relationship between metabolic syndrome and eGFR used the old glucose cutoff of $\geq 6.1 \mathrm{mmol} / \mathrm{L}$ as a criterion for the metabolic syndrome [2, 5], we re-analyzed our data using this cut-point. There was still no fundamental change in the relationship among metabolic syndrome, insulin resistance and eGFR (data not shown), with the exception that the prevalence of the metabolic syndrome diagnosis decreased from 42 to $34 \%$ using the higher glucose cut-point.

\section{Discussion}

Previous studies have suggested an association between the diagnosis of the metabolic syndrome and an increased risk for developing CKD. In this study, we also observed an association between the diagnosis of the metabolic syndrome and a modest decline in eGFR. Interestingly, of all the components of metabolic syndrome, we found that only hypertension was significantly associated with an increased risk for CKD. In contrast, insulin resistance, directly measured by the insulin suppression test, was not associated with a decline in eGFR. Our findings indicate that the relationship between metabolic syndrome and kidney function may be explained by risk conferred by individual components of the syndrome, such as hypertension, rather than by insulin resistance.

Our study is different from earlier studies reporting an association between insulin resistance and CKD because these studies used surrogate markers of insulin resistance $[2,5,6]$. The most commonly used marker has been the homeostasis model assessment of insulin resistance (HOMA-IR), which is a calculation based on fasting glucose and insulin. HOMA-IR is a limited surrogate marker because it accounts for $<40 \%$ of the variability as quantified by more direct measures of insulin resistance $[7,25]$. The metabolic syndrome diagnosis has also been used as a surrogate measure of insulin resistance. However, as seen in Table 2, the relationship between metabolic syndrome and insulin resistance is not perfect, with $16 \%$ of the most insulin-sensitive individuals qualifying for the diagnosis of metabolic syndrome. Therefore, it is not entirely surprising to find conflicting results between studies using surrogate markers and direct measures of insulin resistance.

Although we did not find a relationship between insulin resistance and kidney function, our results do support previous findings showing an association between the metabolic syndrome diagnosis and impaired kidney function. The marginal significance levels seen in our study may relate to our smaller sample size compared with those of previous studies, which included 6000 to 10000 subjects [1,2]. The relationship between the metabolic syndrome and CKD likely reflects two major factors known to be associated with CKD initiation or progression. Firstly, individuals with metabolic syndrome are generally older than their counterparts without metabolic syndrome $[1,2]$. Older age is known to be a strong risk factor for CKD [25]. Secondly, each of the components of the metabolic syndrome has been identified to be associated with CKD [26-32]; thus, the diagnosis may reflect additive effects of the individual components on CKD risk. The risk conferred by each component, however, is probably not equal. In our study, hypertension had the strongest association with CKD, which is consistent with findings from other studies [1, 2]. For example, in 10096 nondiabetic participants enrolled in the Atherosclerosis Risk in Communities Study [2], a greater proportion of hypertensive individuals developed CKD compared with individuals with any other single component of the metabolic syndrome (11 versus $<9 \%)$. Notably, hypertensive individuals accounted for $46 \%$ of the total number of individuals who developed $\mathrm{CKD}$; in comparison, individuals with metabolic syndrome diagnosis accounted for $30 \%$ of the total number of individuals who developed CKD.

The strong association between hypertension and CKD is particularly interesting because hypertension is the component of the metabolic syndrome least associated with insulin resistance $[33,34]$. Although insulin-resistant individuals are more likely to be hypertensive compared with insulin-sensitive individuals, only half of hypertensive 
individuals are insulin-resistant (in upper tertile of insulin resistance) [35]. Moreover, using factor analysis to identify related variables among the metabolic syndrome components and insulin resistance, Ferrannini [33] demonstrated that triglyceride and HDL-C concentration but not hypertension cluster with insulin resistance as measured by the hyperinsulinemic euglycemic clamp method.

Our findings highlight two main limitations of the metabolic syndrome diagnosis. Firstly, this diagnosis does not accurately stratify individuals with varying degrees of insulin resistance compared to direct measures of insulin sensitivity. Secondly, although the metabolic syndrome diagnosis may predict cardiovascular disease and CKD, the predictive power of this diagnosis is likely driven by individual components of the syndrome such as glucose intolerance, dyslipidemia and hypertension. Thus, the metabolic syndrome diagnosis does not convey additional prognostic information when compared to that provided by the sum of its individual components. Due to these limitations, the clinical utility of the metabolic syndrome has been recently debated [36-38].

In comparison to previous studies employing direct measures of insulin sensitivity to evaluate the association between insulin resistance and kidney function [9-11], our study differed significantly. These prior studies examined insulin resistance in individuals with significant kidney disease, and although a relationship between insulin resistance and kidney function was identified, it is not clear whether insulin resistance is a consequence or a cause of overt kidney disease. For example, DeFronzo et al. [9] used the euglycemic clamp technique to evaluate insulin sensitivity in patients with end-stage renal disease $(n=10)$ and reported lower degrees of insulin sensitivity compared to that of controls $(n=8)$. Kobayashi et al. [10] also reported lower mean insulin sensitivity in patients diagnosed with CKD $(n=29)$ compared with controls $(n=10)$. Within the group of individuals with kidney disease, there was a modest association between serum creatinine concentration and insulin sensitivity $(r=-0.449)$. Finally, a recent study suggested a linear association between insulin sensitivity and eGFR in older men $(n=1070)$ with a mean age of 71 and a cystatin-based eGFR of $61.5 \mathrm{~mL} / \mathrm{min} / 1.73 \mathrm{~m}^{2}$ [11]. The relationship was modest, with a large (one unit) decrease in insulin sensitivity correlating with a small $(0.85-$ $1.19 \mathrm{~mL} / \mathrm{min} / 1.73 \mathrm{~m}^{2}$ ) decline in eGFR. Collectively, these studies suggest a relationship between insulin resistance and overt kidney disease but it is not clear what is the nature of this relationship. To circumvent the potential confounding factor of the presence of advanced kidney disease contributing to insulin resistance, we evaluated the association between kidney function and insulin resistance in a population prior to overt kidney disease and found no meaningful relationship.

There are several limitations to our study. Firstly, we examined a cross-sectional study of individuals with a low prevalence of CKD. Nevertheless, the prevalence of CKD estimated by the MDRD equation was higher than that found in the NHANES population without diabetes (4.2\% in this study compared with $1.9 \%$ in NHANES [5]). Secondly, although we studied a population that was much larger than those of previous studies using direct measures of insulin resistance $[9,10]$, our sample size was still smaller than those of epidemiological studies using indirect measures of insulin resistance [1,2]. Therefore, we could have missed smaller associations between insulin resistance and kidney function that would have been apparent with larger samples. Thirdly, we evaluated only the association between insulin resistance and kidney function and therefore cannot exclude the possibility that insulin resistance could influence the future development of kidney disease. Finally, to assess kidney function, we did not evaluate other markers of kidney disease such as microalbuminuria in our study. We primarily used estimation equations for glomerular filtration rate, which are surrogates for direct measurements of glomerular filtration. To address this inherent limitation, we did employ two newer equations, CKD-EPI and Mayo, which have been specifically developed for calculating eGFR in healthier populations. Although the prevalence of CKD varied with the method used to calculate eGFR, the direction of the association between the metabolic syndrome diagnosis and eGFR remained similar to and consistent with past studies.

In conclusion, we analyzed the association among insulin resistance, the metabolic syndrome and eGFR in 574 relatively healthy individuals and found no meaningful correlation between insulin resistance and eGFR. While decreased kidney function may be more common in individuals with the metabolic syndrome than in those without it, our findings argue against the role of insulin resistance as a common factor for CKD.

Acknowledgements. Funding support was provided by National Institutes of Health research grants MH079114 (S.H.K.) and DK0773487 (A.C.P.).

Conflict of interest statement. None declared.

\section{References}

1. Chen J, Muntner P, Hamm LL et al. The metabolic syndrome and chronic kidney disease in U.S. adults. Ann Intern Med 2004; 140: 167-174

2. Kurella M, Lo JC, Chertow GM. Metabolic syndrome and the risk for chronic kidney disease among nondiabetic adults. J Am Soc Nephrol 2005; 16: 2134-2140

3. Chen J, Gu D, Chen CS et al. Association between the metabolic syndrome and chronic kidney disease in Chinese adults. Nephrol Dial Transplant 2007; 22: 1100-1106

4. Luk AO, So WY, Ma RC et al. Metabolic syndrome predicts new onset of chronic kidney disease in 5,829 patients with type 2 diabetes: a 5-year prospective analysis of the Hong Kong Diabetes Registry. Diabetes Care 2008; 31: 2357-2361

5. Chen J, Muntner P, Hamm LL et al. Insulin resistance and risk of chronic kidney disease in nondiabetic US adults. J Am Soc Nephrol 2003; 14: 469-477

6. Landau M, Kurella-Tamura M, Shlipak MG et al. Correlates of insulin resistance in older individuals with and without kidney disease. Nephrol Dial Transplant 2011; Epub ahead of print

7. Kim SH, Abbasi F, Reaven GM. Impact of degree of obesity on surrogate estimates of insulin resistance. Diabetes Care 2004; 27 : 1998-2002

8. Yeni-Komshian H, Carantoni M, Abbasi F et al. Relationship between several surrogate estimates of insulin resistance and quantification of insulin-mediated glucose disposal in 490 healthy nondiabetic volunteers. Diabetes Care 2000; 23: 171-175 
9. DeFronzo RA, Tobin JD, Rowe JW et al. Glucose intolerance in uremia. Quantification of pancreatic beta cell sensitivity to glucose and tissue sensitivity to insulin. J Clin Invest 1978; 62: 425-435

10. Kobayashi S, Maesato $\mathrm{K}$, Moriya $\mathrm{H}$ et al. Insulin resistance in patients with chronic kidney disease. Am J Kidney Dis 2005; 45: 275-280

11. Nerpin E, Riserus U, Ingelsson E et al. Insulin sensitivity measured with euglycemic clamp is independently associated with glomerular filtration rate in a community-based cohort. Diabetes Care 2008; 31 : $1550-1555$

12. U.S. Department of Health and Human Services PHS. NHANES III Anthropometric Procedures Video. Washington, DC: U.S. Government Printing Office, 1996

13. Rule AD, Larson TS, Bergstralh EJ et al. Using serum creatinine to estimate glomerular filtration rate: accuracy in good health and in chronic kidney disease. Ann Intern Med 2004; 141: 929-937

14. Levey AS, Stevens LA, Schmid CH et al. A new equation to estimate glomerular filtration rate. Ann Intern Med 2009; 150: 604-612

15. Pei D, Jones CN, Bhargava R et al. Evaluation of octreotide to assess insulin-mediated glucose disposal by the insulin suppression test. Diabetologia 1994; 37: 843-845

16. Greenfield MS, Doberne L, Kraemer F et al. Assessment of insulin resistance with the insulin suppression test and the euglycemic clamp. Diabetes 1981; 30: 387-392

17. Expert Panel on Detection, Evaluation, and Treatment of High Blood Cholestrol in Adults (Adult treatment panel III). Executive Summary of The Third Report of The National Cholesterol Education Program (NCEP). JAMA 2001; 285: 2486-2497

18. Grundy SM, Cleeman JI, Daniels SR et al. Diagnosis and management of the metabolic syndrome: an American heart Association/National heart, Lung, and blood Institute Scientific statement. Circulation 2005; 112: 2735-2752

19. Maschio G, Alberti D, Janin G et al. Effect of the angiotensinconverting-enzyme inhibitor benazepril on the progression of chronic renal insufficiency. The Angiotensin-Converting-Enzyme Inhibition in Progressive renal Insufficiency Study Group. N Engl J Med 1996; 334: 939-945

20. Randomised placebo-controlled trial of effect of ramipril on decline in glomerular filtration rate and risk of terminal renal failure in proteinuric, non-diabetic nephropathy. The GISEN Group (Gruppo Italiano di Studi Epidemiologici in Nefrologia). Lancet 1997; 349: 1857-1863

21. Brenner BM, Cooper ME, de Zeeuw D et al. Effects of losartan on renal and cardiovascular outcomes in patients with type 2 diabetes and nephropathy. $N$ Engl J Med 2001; 345: 861-869

22. Lewis EJ, Hunsicker LG, Clarke WR et al. Renoprotective effect of the angiotensin-receptor antagonist irbesartan in patients with nephropathy due to type 2 diabetes. New Engl J Med 2001; 345: $851-860$
23. Tonelli M, Isles C, Craven $\mathrm{T}$ et al. Effect of pravastatin on rate of kidney function loss in people with or at risk for coronary disease. Circulation 2005; 112: 171-178

24. Shepherd J, Kastelein JJ, Bittner V et al. Effect of intensive lipid lowering with atorvastatin on renal function in patients with coronary heart disease: The treating to New Targets (TNT) study. Clin J Am Soc Nephrol 2007; 2: 1131-1139

25. Finucane FM, Luan J, Wareham NJ et al. Correlation of the leptin: adiponectin ratio with measures of insulin resistance in non-diabetic individuals. Diabetologia 2009; 52: 2345-2349

26. Buckalew VM Jr., Berg RL, Wang SR et al. Prevalence of hypertension in 1,795 subjects with chronic renal disease: the modification of diet in renal disease study baseline cohort. Modification of Diet in Renal Disease Study Group. Am J Kidney Dis 1996; 28: 811-821

27. Ejerblad E, Fored CM, Lindblad P et al. Obesity and risk for chronic renal failure. J Am Soc Nephrol 2006; 17: 1695-1702

28. Wang Y, Chen X, Song Y et al. Association between obesity and kidney disease: a systematic review and meta-analysis. Kidney Int 2008; 73: 19-33

29. Fox CS, Larson MG, Leip EP et al. Glycemic status and development of kidney disease: the Framingham Heart Study. Diabetes Care 2005; 28: 2436-2440

30. Hunsicker LG, Adler S, Caggiula A et al. Predictors of the progression of renal disease in the Modification of Diet in Renal Disease Study. Kidney Int 1997; 51: 1908-1919

31. Muntner P, Coresh J, Smith JC et al. Plasma lipids and risk of developing renal dysfunction: the atherosclerosis risk in communities study. Kidney Int 2000; 58: 293-301

32. de Boer IH, Katz R, Fried LF et al. Obesity and change in estimated GFR among older adults. Am J Kidney Dis 2009; 54: 1043-1051

33. Ferrannini E, Balkau B, Coppack SW et al. Insulin resistance, insulin response, and obesity as indicators of metabolic risk. J Clin Endocrinol Metab 2007; 92: 2885-2892

34. Meigs JB, D'Agostino RB Sr., Wilson PW et al. Risk variable clustering in the insulin resistance syndrome. The Framingham Offspring Study. Diabetes 1997; 46(10):1594-1600

35. Lima NK, Abbasi F, Lamendola C et al. Prevalence of insulin resistance and related risk factors for cardiovascular disease in patients with essential hypertension. Am J Hypertens 2009; 22: 106-111

36. Kahn R, Buse J, Ferrannini E et al. The metabolic syndrome: time for a critical appraisal: joint statement from the American diabetes association and the European association for the study of diabetes. Diabetes Care 2005; 28: 2289-2304

37. Reaven GM. The metabolic syndrome: requiescat in pace. Clin Chem 2005; 51: 931-938

38. Grundy SM. Does the metabolic syndrome exist? Diabetes Care 2006; 29: 1689-1692; discussion 1693-1696

Received for publication: 16.5.11; Accepted in revised form: 21.7.11 